DOI: http://dx.doi.org/10.35381/racji.v4i7.395

\title{
La ley de infogobierno en el contexto tecnológico de la gestión pública
}

The infogobierno law in the technological context of public management

\author{
Nathaly Serrano de Barrios \\ pnfi.iuty@gmail.com/nathalyaracuy@gmail.com \\ Universidad Politécnica Territorial de Yaracuy Arístides Bastidas \\ Venezuela \\ https://orcid.org/0000-0003-1422-8260
}

Recibido: 1 de mayo de 2019

Aprobado: 14 de junio de 2019

\section{RESUMEN}

La Ley de Infogobierno surge con el propósito de contribuir a una gestión gubernamental eficiente basada en la tecnología. Fue promulgada en 2013, Gaceta No. 40.274, derivada de la evolución del Decreto 3.390 (2004) y el Decreto con fuerza de Ley de Acceso e Intercambio Electrónico de Datos, Información y Documentos entre los Órganos y Entes del Estado (2012), ambos derogados por la ejecución de dicha entidad legal destinada a integrar información en un ecosistema de gobierno electrónico, a través del binomio del interés público de la ciencia, la tecnología y la innovación del artículo 110 de la Carta Magna (CRBV, 1999) y los servicios vinculantes entre el Poder Público y el Poder Popular. La investigación de tipo descriptivo con diseño documental, tiene como objetivo describir una síntesis teórica del marco legal tecnológico que sustenta la gestión de las políticas públicas en la práctica, fragmentando estadíos de dominación socio-tecnológica.

Descriptores: Gobierno electrónico; Información; Gestión; Políticas públicas; Tecnología.

\section{ABSTRACT}

The Law of Infogovernment emerges with the purpose of contributing to efficient government management based on technology. It was promulgated in 2013, Gazette No. 40,274, derived from the evolution of Decree 3,390 (2004) and the Decree with force of Law on Access and Electronic Exchange of Data, Information and Documents between the Bodies and State Entities (2012), both repealed by the execution of said legal entity aimed at integrating information in an electronic government ecosystem through the binomial of the public interest of science, technology and innovation of article 110 of the 
Magna Carta (CRBV, 1999) and binding services between the Public Power and the Popular Power. Descriptive type research with documentary design is aimed at telling a theoretical synthesis of the technological legal framework that underpins the management of public policies in practice, fragmenting stadiums of socio-technological domination.

Descriptor: Electronic government; Information; Management; Public policy; technology.

\section{INTRODUCCIÓN}

El escaso uso de la tecnología en los procesos internos de la gestión pública aunada a una visión aislada y con baja calidad de servicio al ciudadano convocó en la Carta Magna la necesidad de contemplarla como interés público en sistémica armonía con la ciencia y la innovación como elementos claves de desarrollo.

Es así como con la promulgación de la Constitución de la República Bolivariana de Venezuela (1999) el Estado venezolano inicia un transitar de difusión, dotación y campaña de apropiación tecnológica en todo el territorio nacional hacia la soberanía tecnológica en función de la ruptura de la dominación, logro de la independencia y autodeterminación.

A partir de la Ley Orgánica de Telecomunicaciones (LOTEL, 2000) en la primera edición, se afianza el interés global por la tecnología y la amplitud de servicios en las áreas comunicativas. El enfoque primario se dirigió a la infraestructura, mobiliario, equipos (hardware), servicio de Internet. Para luego avocarse a los procesos de alfabetización tecnológica hacia la apropiación digital.

El ejercicio de las políticas tecnológicas componentes de las políticas públicas venezolanas a partir de la nueva Constitución, le concede un giro a la gestión pública incidiendo en la transformación del contexto tecnológico, organizacional y social primariamente.

En el tecnológico, las organizaciones estaban atadas a software privativo, inclusive el denominado coloquialmente: pirata. Copia de software ilegal en virtud de los altos costos 
de las licencias o presupuestos restringidos para hacerle frente a tarifas de uso del software por cada estación de trabajo.

A nivel organizacional, los procesos administrativos se desarrollaban en su mayoría de forma manual o utilizando herramientas ofimáticas. Los pocos sistemas existentes habían sido desarrollados en software privativo con débil posibilidad de escalar y con las limitaciones del mismo. Por otra parte, los ciudadanos y ciudadanas se debían dirigir hasta la sede del ente público para solicitar los servicios y, posteriormente, retornar a buscar los documentos en cuestión.

Con el despliegue tecnológico geopolítico, el Poder Popular empezó a acceder a través de internet al portal de alguna entidad oficial para realizar un registro, solicitar un trámite, sin necesidad de trasladarse a la entidad física.

A pesar de esos logros, se mantienen a casi veinte años de la Constitución, rasgos de oposiciones a los sistemas nacionales y dependencia tecnológica, nudos críticos de la Ley de Infogobierno ante el apego a recursos privativos y la conformación de sistemas interconectados.

La presente investigación se justifica a nivel legal dado la puesta en práctica de la Ley a través de los sistemas gubernamentales y los diversos portales Web. En el aspecto social, se ampliará en las posibilidades del conocimiento libre, la protección y el acceso a la información. En el ámbito de políticas públicas nacionales se hace letra activa la Ley que impulsa el gobierno electrónico y el sector de tecnologías de información libres.

Tal realidad vislumbra el objetivo del presente artículo: describir una síntesis teórica del marco legal tecnológico para la gestión de las políticas públicas en la práctica, fragmentando estadios de dominación socio-tecnológica. Bajo una investigación descriptiva con diseño teórico documental de acuerdo a Ramírez (1999) con énfasis en los aspectos legales de la Ley de Infogobierno como política pública de enlace entre el Poder Público con el Poder Popular en el tejido social venezolano. 


\section{Tecnología en la Gestión Pública}

La tecnología en la gestión pública tiene su génesis legal en el Artículo 110 de la Constitución de la República Bolivariana de Venezuela (1999), al otorgar interés público a la ciencia, tecnología e innovación como factores de impulso productivo nacional.

La tecnología se basa en la capacidad instalada de la institución, la cual incluye tanto recursos tecnológicos como humanos y materiales. Entendiéndose por tecnología aquellas actividades científicas aplicadas para crear o modificar elementos materiales y culturales de nuestro entorno natural o artificial (Bunge, 2005)

Su importancia radica en ser mediador en la administración pública de la información comunicación y conocimiento de los procesos institucionales con el fin de otorgarle fluidez, veracidad y velocidad, así como posibilidad de estadísticas, resguardo de datos y memoria institucional.

Constituye entonces, un recurso estratégico para la gestión transparente en todos los niveles: municipal, estatal y nacional, con la complejidad de responder en las diversas aristas: educación, salud, administración, tributos y documentación (Mayorga y Galaviz, 2017).

La multiplicidad de servicios de los entes públicos y la distancia existente entre ellos es superada por la tecnología, las conexiones inalámbricas o satelitales accesibles. Los servicios tecnológicos son determinantes en una gestión pública orientada al bienestar social. Internet está para acercar distancias, personas y sucesos.

"El internet y las redes sociales propician la igualdad social, la conectividad individual y la interlocución entre personas, sociedades y culturas. La democracia y la protección de los derechos humanos se han fortalecido gracias a la Revolución Tecnológica" (Grisanti, 2015:p.295)

En lo planteado por Grisanti, se evidencia a la tecnología factor clave en el quiebre radical de las relaciones tradicionales entre las personas con el Estado. Derivado por las múltiples formas de comunicación e información digital, nuevos órdenes, nuevos signos, nuevas interacciones humanas. Le corresponde al Estado conectarse con la revolución 
tecnológica como forma de interacción ciudadana, evitando el rezago tecnológico y adoptando otras vías de llegar a las comunidades más remotas.

El aceleramiento de la prestación de servicios públicos centrales y locales pueden ayudarse a través de la tecnología (McKinsey, 2016 en Shava and Hofisi, 2017). Es así como progresivamente se añadieron servicios digitales a los ciudadanos y ciudadanas incidiendo en la apropiación tecnológica, expandiendo la denominada revolución tecnológica a revolución social.

En un proceso introspectivo, son revoluciones de mutua interacción, los usuarios de tecnología exigen nuevos servicios y los creadores de tecnología compiten por la fidelidad de los clientes y continuidad de transacciones a favor de sus productos, con elementos diferenciadores, funciones sencillas y automáticas. El mercado tecnológico es muy llamativo para los usuarios, sin distinción de edad, género o religión.

Así fue asumido por el Estado venezolano al crear desde el año 2000 los Infocentros con el fin ejercer el principio de inclusión e inspirar el desarrollo socio tecnológico de las comunidades, fortalecido con la creación del Ministerio del Poder Popular de Ciencia, Tecnología e Innovación (MPPCTI, 1999) al instalar más de 2.300 centros de acceso a nivel nacional.

A comienzos de milenio, se aprueba la Ley de Telecomunicaciones (2000) con actualización en el año 2016, dada la necesidad de regular lo concerniente a la telecomunicación, redes y comunicación satelital.

La gestión pública tiene en la tecnología aliados de las políticas públicas en cuanto a la inclusión social y digital, tomando en cuenta a todas las venezolanas y venezolanos en su virtud de ser humano independientemente del lugar recóndito de habitación. Visibilizar a los excluidos durante el transcurso del proceso formativo de software libre en un Infocentro en comunidades remotas, fue innovador en el año 2000 y se hizo natural desde la capital hasta el extremo del Amazonas.

El Estado, en su afán de fortalecer las políticas públicas tecnológicas, emite el Decreto 825 (2000) correspondiente al Acceso y el uso de Internet como política prioritaria para 
el desarrollo cultural, económico, social y político de la República Bolivariana de Venezuela. Fortalecido a través de la distribución de Infocentros a nivel nacional y la libertad regulada del espectro de telecomunicaciones, favoreció la comunicación, envío y recepción de mensajes y datos intra e interinstitucional con el fin de aumentar la velocidad de los trámites, ahorro de tiempo, energía y materiales de oficina en armonía absoluta con la naturaleza.

En consecuencia, sobreviene el Decreto con fuerza de Ley sobre mensajes de datos y firmas electrónicas (2001) cuyo objeto está dirigido al reconocimiento de la validez, eficacia y valor jurídico de la Firma Electrónica, el Mensaje de Datos y a toda información inteligible en formato electrónico tan necesario en los trámites intergubernamentales digitales.

Para el 2001, acontece la publicación del Decreto con Rango y Fuerza de Ley de Ciencia, Tecnología e Innovación (LOCTI, 2001), plataforma de la Ley Orgánica de Ciencia, Tecnología e Innovación, aprobada por la Asamblea Nacional en 2005 reformada en el 2010.

La documentación legal mencionada sostiene la tecnología e innovación de la gestión pública, concebida ella desde la corresponsabilidad, la participación protagónica y la consulta pública. Nace de ese modo, tras una consulta colectiva dispuesta en la actualización de SITEAL (2018), el Plan Nacional de Telecomunicaciones, Informática y Servicios Postales (PNTlySP, 2007-2013) cuyo énfasis es reconocer la comunicación como un Derecho Humano a través de las líneas generales de uso masivo de las TIC, la soberanía e independencia tecnológica, transformación del Estado, uso y aplicación de las TIC y los servicios postales como dimensiones de habilitación de desarrollo y modelo comunicacional inclusivo.

Otra evidencia de consulta pública es el Plan Nacional Simón Bolívar llamado el primer Plan socialista (2007-2013), el Plan Nacional de Ciencia Tecnología e Innovación la Ley Orgánica del Plan de la patria Segundo Plan Socialista de Desarrollo Económico y Social 
de la Nación 2013-2019. (2013) y la Ley del Plan de la Patria Tercer Plan socialista (20192025) y el Plan Nacional de Ciencia Tecnología e Innovación (PNCTI 2005-2030) En tal sentido, la República Bolivariana de Venezuela (2016), de acuerdo a Presentación Nacional Voluntaria (PNV) ante El Foro Político de Alto Nivel Sobre Desarrollo Sustentable (FPAN) de Naciones Unidas reporta el reconocimiento a la Fundación Infocentro otorgado por la UNESCO en el año 2011 por el Programa Nacional de Alfabetización Tecnológica (PNAT). Exaltando las políticas públicas dirigidas a socializar el conocimiento y las tecnología a sectores de escasos recursos como instrumento de apropiación y divulgación socio tecnológica del acontecer comunitario, regional y nacional. Con posibilidades de usar los servicios digitales y participar en las consultas nacionales.

Un ejemplo de ello lo conformó la invitación nacional a participar activamente en las deliberaciones asociadas a la Ley de Telesalud donde la ciudadanía abrió espacio en la Red Internet mediante una Web editable denominada Wiki fue titulada "wiki legislación" en la cual redactaban sus opiniones e inquietudes relativas a los artículos de la propuesta de la Ley de Telesalud en medio de un debate constructivo digital. No obstante, para Palm y Petrizzo (2010) la wiki debió calificarse como "wiki deliberación" dada la naturaleza y dinámica de la misma.

Los autores mencionados basan su planteamiento en las características propias de la deliberación pública con una consulta nacional de leyes dado los procesos de construcción, deconstrucción y reconstrucción de textos legales a través de asambleas digitales asíncronas en un lapso de tiempo determinado por el poder público.

Las posibilidades de la tecnología en la gestión pública son múltiples y diversas. Sin embargo no se debe dejar arropar por sus evidentes ventajas las cuales ceden a desventajas sin la dirección, finalidad y protección pertinente.

Khurana (2019) expresa "Su propósito solo puede entenderse completamente por sus fines funcionales dentro de la estructura más amplia. Las tecnologías se desarrollan a 
partir de ciertas intenciones, se implementan a través de ciertos arreglos institucionales e incentivan ciertas formas de comportamiento" (p.7)

En fin, el impacto de la tecnología es mucho más amplio que la estructura en la cual está inserta, impacta las personas, los procesos, la distribución de recursos y cultura institucional. De allí la importancia del equipo de gestión pública ante la filosofía de valorar los datos e información gubernamental, como gestores de información de los ciudadanos y ciudadanos que confiaron en ellos como autoridades locales y nacionales. Quienes además, deben garantizar la confidencialidad de los datos personales y de tributo al gobierno en los procesos de intercambio con las otras entidades públicas en función de minimizar los trámites administrativos y potenciar la Ley de Infogobierno.

\section{Ley de Infogobierno y Gobierno Electrónico}

La ley de Infogobierno ejecutada en el año 2013, refiere la información gubernamental como interés público a la par de la adopción de las tecnologías libres como filosofía de libertad inmerso en el plan político socialista nacional. La esencia es mejorar la gestión del Poder Público para atender las demandas de los servicios que por derecho se deben al Poder Popular.

Esta Ley surgió con el propósito de ofrecer a los ciudadanos y ciudadanas, servicios de información integrales a partir del enfoque de interoperabilidad entre las diferentes instituciones del estado hacia la integración promoviendo las tecnologías de información libre en su acrónimo TIL.

Con esta Ley se benefician todos y cada uno de los ciudadanos venezolanos que interaccionan con la tecnología. La finalidad investigativa es promover el espíritu sistémico, emprendedor, social, académico y tecnológico hacia la sinergia necesaria de las bondades de la apropiación tecnológica, la calidad de servicio en la gestión pública y la apreciación de las políticas públicas en el quehacer diario los venezolanos y venezolanas. 
En 84 artículos, establece los principios bases y lineamientos dirigidos al servicio público mediado por la tecnología como derecho del pueblo. Sustentado ello por las deliberaciones de las diferentes fuerzas vivas del sector de telecomunicaciones e informática a nivel nacional, representantes del gobierno y de la administración pública. Su base conceptual nucleica es la interoperabilidad, admitida como la capacidad de uso e intercambio íntegro de datos, información y documentos de los ciudadanos y ciudadanas entre los diversos sistemas de información de las entidades de estado.

El Grupo de Trabajo para la Interoperabilidad, de la Asociación Francófona de Usuarios de Software Libre citado por Kruger (2017): la define como: "La interoperabilidad es la capacidad que tiene un producto o un sistema, cuyas interfaces son totalmente conocidas, para funcionar con otros productos o sistemas existentes o futuros y eso sin restricción de acceso o de implementación." (p.1)

Las normas técnicas, semánticas y organizacionales deben ser compartidas por los entes a involucrados para una transferencia de datos e información fluida. Su importancia radica en posibilitar el compartir conocimiento, el desarrollo de la administración y el gobierno electrónico.

Grosvald (2005) citado por Correa y Criollo (2010), refieren como Gobierno Electrónico aquel que:

Incluye todas aquellas actividades basadas en las nuevas tecnologías informáticas, en particular Internet, que el Estado desarrolla para aumentar la eficiencia de la gestión pública, mejorar los servicios ofrecidos a los ciudadanos y proveer a las acciones del gobierno de un marco mucho más transparente que el actual, tanto en las aplicaciones internas como las externas de las tecnologías de la información y las comunicaciones (TIC) en el sector público. (p.41)

Dichos sistemas deben estar desarrollados en software libre definido en la misma Ley como los programas con licencia libre para usarlo con cualquier propósito reconociendo la autoría y protegiendo el derecho moral de sus creadores. Fomenta el trabajo colaborativo y la libertad de uso. 
Ejemplo activo de interoperabilidad y la Ley de Infogobierno es el sistema Patria accesible a través del carnet de la patria instrumento generado por el gobierno venezolano para conceder beneficios sociales a la población mediante los programas sociales activos. "Esta herramienta busca por medio del registro personalizado, englobar todos los datos socioeconómicos del portador. A fin de que sean otorgados los planes sociales que desarrolla el ejecutivo nacional, con prioridad a los más necesitados" (infogobierno.net) El sistema Patria agrupa en un solo sistema el perfil laboral, social y económico de los ciudadanos y ciudadanas registradas a objeto de realizar acompañamiento a los programas sociales gubernamentales obteniendo información desde el número de cédula de identidad del usuario en conjunto con el código del carnet de la patria. Integrando en un solo sistema de información datos del Sistema de Identificación, Poder Electoral y laboral si es funcionario público.

El perfil también involucra los datos del grupo familiar. Eso es similar en cada uno de los portales del gobierno. Es importante resaltar el hecho que los datos e información son institucionales, gubernamentales. Su objeto precisa dar respuestas a los ciudadanos y los entes de gobierno superiores. Debe ser íntegra, confiable y estar disponible en los órganos y entes del ámbito de aplicación de la Ley, protegida y con restricción de acceso, lejos de usos personales de lo contrario está penado por las leyes correspondientes.

Es válido destacar lo contemplado en dicho documento legal ante la resistencia de uso de software libre persistente en algunos entes estatales, se ofreció diez meses de plazo como lapso de tiempo para su vigencia formal. En función de la adaptación o migración si aplicaba, de los programas informáticos a software libre y estándares abiertos, ya no como prioridad sino como obligatoriedad con posibilidades de excepciones establecidas u autorizadas debidamente por la Comisión Nacional de Tecnologías de Información (CONATI).

Conforme a lo anterior, la Ley avista régimen sancionatorio para los funcionarios, funcionarias, servidores y servidoras públicas, así como excepciones, certificaciones y contribuciones por la utilización de software privativo. Promueve el uso general de las 
tecnologías de información libre en el marco del gobierno electrónico nacional, vitrina digital de los servicios y derechos del soberano venezolano.

La convocatoria de uso de la tecnología libre pasa por disponer de un portal web y colocar al alcance de los ciudadanos y ciudadanas lo servicios pertinentes. Adicionalmente, revisar procesos normas y procedimientos en su dinámica, ante los cambios y las demandas del entorno social, político y los recursos tecnológicos disponibles.

Massal y Sandoval (2010) mencionan como posibles obstáculos para acceder al gobierno electrónico, los vinculados a las limitaciones técnicas, calidad de servicio de internet, la disponibilidad de cuenta bancaria y la habilidad en el uso de la tecnología. Superados muchos de ellos en Venezuela por las políticas y programas públicos de acceso y apropiación tecnológica, así como otros servicios de cuenta bancaria. A pesar de la coyuntura y los ataques deliberados a los servicios públicos, sobretodo, el de energía eléctrica.

Perozo y Marín (2019) indican "Venezuela, se ubica en un nivel calidad bajo de servicios en línea, de acuerdo a esto, se infiere que las dimensiones infraestructura de telecomunicaciones y capital humano supera el alcance y la calidad de los servicios en línea" (p.101). En tal sentido la infraestructura tecnológica funcional, accesible y actualizada es factor crítico de éxito para el gobierno electrónico eficiente.

Amerita por tanto, la modernización de las dotaciones tecnológicas, la masificación de la Ley de Infogobierno y la consolidación del gobierno electrónico en reingeniería de los procesos informacionales al margen del presente documento legal, con el fin de reemplazar y optimizar los programas informáticos a las nuevas demandas, siempre con el norte de la independencia tecnológica y el deseo de ser País potencia, delineado en la Ley del Plan de la Patria (2019-2025).

La información determina el pasado, presente y futuro institucional. De allí su importancia y necesidad de resguardo pautada en los productos informáticos a través de las opciones de respaldar, restaurar y la bitácora para la auditoría respectiva de las transacciones y acciones electrónicas. Previsto ello en la Ley de Infogobierno en el interés público y 
estratégico de las tecnologías de información, el principio de seguridad y las certificaciones electrónicas, entre otras dirigidas a la optimización de la limpidez y validez de la gestión pública.

La promoción de las tecnologías libres debe asumirse en primera instancia, a lo interno de la gestión pública local o nacional, en los procesos propios del funcionamiento de dicha entidad: intrainstitucional. Dicha entidad del Estado en relación con otras entidades estatales deben establecer un diálogo comunicacional mediado por la tecnología, ese diálogo entre procesos de diversas instituciones se transforma en interinstitucional.

Ese diálogo bidireccional tecnológico es una analogía del ejercicio de la interoperabilidad de los sistemas de cada entidad de estado como estrategia de consolidación del gobierno electrónico, con desarrollo y uso de tecnologías libres desde lo local, autóctono, por y para venezolanos y venezolanas, hacia la soberanía tecnológica y la independencia Nacional, caracterizado ello en el Primer Objetivo Histórico del Plan de la Patria (20192025).

La interoperabilidad entre entes gubernamentales involucra a la universidad como otra fuente de conocimiento e investigación que ante el gobierno electrónico, debe conectarse en un círculo virtuoso de reingeniería comunicacional de procesos y conocimientos. González de H., E. y Isea J. (2019) señalan:

El campo social en el cual se inserta la universidad, es un espacio de agenciamiento que comporta componentes heterogéneos de orden biológico, social, tecnológico, gnoseológico e imaginario de múltiples rizomas que la investigación descifra, aunque sea en parte, para producir conocimientos, devenires, y proponer alternativas a las demandas generadas por sus integrantes, internas, y su entorno (externas). (p.336)

La agenda común entre el gobierno electrónico y la universidad es el encargo social de ambos, en la cual la investigación es el eje conectivo que vinculado con la tecnología, invitan a la heterogeneidad del saber, a la pluralidad cognitiva y práctica, la interoperabilidad de los conocimientos y la interconexión de sistemas humanos ávidos de transparencia y calidad gubernamental independiente. Esa es la esencia de la Ley de Infogobierno, fundamento del gobierno electrónico transparente, eficiente y oportuno en 
el marco de Políticas Públicas soberanas en resistencia absoluta a la dominación socio tecnológica.

\section{CONCLUSIONES}

La Ley de Infogobierno contribuye al proceso de transformación socio tecnológica Nacional desde los principios de inclusión, deberes gubernamentales y soberanía tecnológica, ejemplo de ello es el Sistema Patria, el cual integra el perfil de los venezolanos y venezolanas como estrategia para la Protección social del gobierno a través de bonos asignados por el carnet patria en alianza con tecnología libre vanguardista.

La Ley de Infogobierno asume la información como un elemento vital en el desarrollo de las actividades de gobierno para mejorar la gestión pública y los servicios a los ciudadanos, estableciendo las bases, principios y lineamientos del uso de las tecnologías de información libre, tanto en el Poder Público como en el Poder Popular.

La población beneficiada está formada por cada venezolano y venezolana usuario directo e indirecto de las tecnologías de información libre. Cada ciudadano sin distinción de edad, profesión u oficio, en fin, todos y todas sin exclusión.

A pesar de las Leyes, hay usuarios institucionales persistentes en la negación del uso de software libre, obviando los esfuerzos cognitivos, gubernamentales y académicos desplegados hacia la apropiación de las tecnologías de información libres.

La Ley de Infogobierno trasciende el ámbito socio tecnológico para ser eje geoestratégico del uso de las tecnologías de información libre a nivel nacional.

El ejercicio de la Ley propicia la infotransformación en el centro de las entidades de gestión pública, fragmentando estadios sociales y tecnológicos de dominación cuando la población accede a servicios electrónicos del estado desde su hogar o Infocentro más cercano, logrando cumplir con sus deberes y disfrutando de sus derechos en formato electrónico. 


\section{REFERENCIAS CONSULTADAS}

1. Bunge, M. (2005) La ciencia. Su método y su filosofía, edición en línea, en www.canariastelecom.com/personales/carlos.clavijo/ebooks/bunge_ciencia.pdf

2. Carnet de la patria: ¿Cómo funciona?. https://infogobierno.net/carnet-de-la-patria/

3. CONATEL (2014). Ley de Infogobierno impulsará uso de las tecnologías libres. Disponible en: http://www.conatel.gob.ve/ley-de-infogobierno-impulsara-uso-delas-tecnologias-libres/

4. Constitución de la República Bolivariana de Venezuela 1999. Gaceta Oficial de la República Bolivariana de Venezuela, 5453 (Extraordinario) marzo 24, 2000

5. Correa Morocho y Criollo Gonzáles:(2010) Gobierno electrónico para la modernización de la administración pública, Edición electrónica gratuita. Texto completo en www.eumed.net/libros/2010e/846/

6. Decreto 3.390 (2004) Gaceta oficial № 38.095. Diciembre 28 de 2004

7. Decreto 825 (2000) (Ley sobre el Acceso y Uso de Internet). Gaceta Oficial № 36.955 mayo 22,2000

8. González de H., E. y Isea J. (2019). La Desterritorilización y el Agenciamiento de los Conocimientos. Una invocación urgente a la investigación universitaria en la postmodernidad. Revista Arbitrada Interdisciplinaria KOINONIA. Año IV. Vol IV. N8. Julio - Diciembre 2019. Hecho el depósito de Ley: FA2016000010.ISSN: 2542-3088

9. Grisanti L. (2015) La revolución tecnológica y la enfermedad holandesa en la compilación Venezuela 2015: Economía, Política y Sociedad Ronald Balza Guanipa Coordinador.-Caracas: Universidad Católica Andrés Bello

10. Khurana, R. (2019) The Fourth Industrial Revolution Will Erode The Power Of Managerial Technocracy. Disponible en https://palladiummag.com/2019/01/22/the-fourth-industrial-revolution-will-erodethe-power-of-managerial-technocracy/

11. Kruger, T. (2017) Interoperabilidad: ¿A qué aspiramos cuando hablamos de ella?. https://www.krugercorp.com/blog-innovacion/interoperabilidad/ 
12. Ley Orgánica del Plan de la patria Segundo Plan Socialista de Desarrollo Económico y Social de la Nación 2013-2019. (2013) Gaceta Oficial de la República Bolivariana de Venezuela No 6.118 Extraordinario, 4 de diciembre de 2013

13. Ley Orgánica del Plan de la patria Tercer Plan Socialista de Desarrollo Económico y Social de la Nación 2019-2025. (2019) Gaceta Oficial Nro. 6.446, abril 8 de 2019

14. Massal, J. and Sandoval, C (2010) Gobierno electrónico. ¿estado, ciudadanía y democracia en internet?: "state, citizenship and democracy on the internet?. anal.polit. [online]. 2010, vol.23, n.68, pp.3-25. ISSN 0121-4705.

15. Mayorga L. y Galaviz G. (2017) La importancia de las tecnologías de información en el sector público Disponible en: https://www.auren.com/es-MX/blog/sectorpublico/2017/01/16/la-importancia-de-las-tecnologias-de-informacion-en-elsector-publico

16. Ley Infogobierno. Gaceta Oficial № 40.274. De octubre 17, de 2013.

17. Ley Orgánica de Ciencia, Tecnología e Innovación (LOCTI) Gaceta Oficial $N^{\circ}$ 38.242, de Agosto de 2005

18. Ley sobre mensajes de datos y firmas electrónicas (2001) Gaceta Oficial № 37.076, Febrero 10, de 2001

19. Ley de Telecomunicaciones (2000) Gaceta. Oficial $N^{\circ}$ 36.970. Junio 12, 2000

20.Palm, F. y Petrizzo, María A. (2010) Ways of Citizen Learning: Political Deliberation on the Internet. en Systems Think and e- Participation: ICT in the governance of society. Pachón-Cordoba, José (ed). United Kingdom: ICI Gobal

21. Perozo R y Marín A. (2019) Incidencias de la Tecnología web 2.0 en el contexto de la gobernanza y la gobernabilidad. Iustitia Socialis. Revista Arbitrada de Ciencias Jurídicas. Año IV. Vol. IV. Nº6. Enero - . Junio 2019. Hecho el depósito de Ley: FA2016000064. ISSN: 2542-3371. FUNDACIÓN KOINONIA (F.K). Santa Ana de Coro, Venezuela

22. Plan Nacional de Ciencia Tecnología e Innovación (2005-2030) http://canaimaeducativo.me.gob.ve/catalogo-unico/contenidos/lecturassugeridas/plan-nacional-de-ciencia-tecnologia-e-innovacion-2005-2030.pdf 
23. Plan Nacional Simón Bolívar 2001-2013. Gaceta Oficial 6.295 Extraordinario 01 de Mayo 2017

24. Ramírez T. (1999). Como hacer un Proyecto de Investigación. . Caracas Editorial Panapo.

25. República Bolivariana de Venezuela (2016) Presentación Nacional Voluntaria (PNV) ante El Foro Político de Alto Nivel Sobre Desarrollo Sustentable (FPAN) de Naciones Unidas. Disponible en https://sustainabledevelopment.un.org/content/documents/10527Venezuela\%20PNV\%20DEFINITIVO\%20JUNIO\%202016\%20\%20DEFINITIVO\%2017062 016\%20(1).pdf

26. SITEAL (2018). Sistema de Información de Tendencias Educativas en América Latina. Venezela.Plan Nacional de Telecomunicaciones, Informática y Servicios Postales (PNTlySP, 2007-2013)

27. Shava, E. and C Hofisi, C. (2017) Challenges and Opportunities for Public Administration in the Fourth Industrial Revolution. Disponible en https://www.researchgate.net/profile/Elvin Shava2/publication/322676701 Chall enges and Opportunities for Public Administration in the Fourth Industrial Revolution/links/5a6865ada6fdcc03e0777a36/Challenges-and-Opportunities-forPublic-Administration-in-the-Fourth-Industrial-Revolution.pdf

\section{REFERENCES CONSULTED}

1. Bunge, M. (2005) Science. His method and philosophy, online edition, at www.canariastelecom.com/personales/carlos.clavijo/ebooks/bunge ciencia.pdf

2. Carnet de la patria: How does it work? https://infogobierno.net/carnet-de-la-patria/

3. CONATEL (2014). Law of Infogovernment will promote the use of free technologies. Available at: http://www.conatel.gob.ve/ley-de-infogobiernoimpulsara-uso-de-las-tecnologias-libres/

4. Constitution of the Bolivarian Republic of Venezuela 1999. Official Gazette of the Bolivarian Republic of Venezuela, 5453 (Extraordinary) March 24, 2000 
5. Correa Morocho and Criollo Gonzáles: (2010) Electronic government for the modernization of public administration, Free electronic edition. Full text at www.eumed.net/libros/2010e/846/

6. Decree 3,390 (2004) Official Gazette No. 38,095. December 28, 2004

7. Decree 825 (2000) (Law on Internet Access and Use). Official Gazette No. 36,955 May 22, 2000

8. González de H., E. and Isea J. (2019). Deterritorilization and Knowledge Agency. An urgent invocation to university research in postmodernity. KOINONIA Interdisciplinary Arbitrated Magazine. Year IV Vol IV. No. 8 July - December 2019.Made the Law deposit: FA2016000010.ISSN: 2542-3088

9. Grisanti L. (2015) The technological revolution and the Dutch disease in the compilation Venezuela 2015: Economy, Politics and Society Ronald Balza Guanipa Coordinator. - Caracas: Andrés Bello Catholic University

10. Khurana, R. (2019) The Fourth Industrial Revolution Will Erode The Power Of Managerial Technocracy. Available at https://palladiummag.com/2019/01/22/thefourth-industrial-revolution-will-erode-the-power-of-managerial-technocracy/

11. Organic Law of the Homeland Plan Second Socialist Economic and Social Development Plan of the Nation 2013-2019. (2013) Official Gazette of the Bolivarian Republic of Venezuela No 6.118 Extraordinary, December 4, 2013

12. Organic Law of the Homeland Plan Third Socialist Economic and Social Development Plan of the Nation 2019-2025. (2019) Official Gazette No. 6,446, April 8,2019

13. Massal, J. and Sandoval, C (2010) Electronic government. ¿State, citizenship and democracy on the internet ?: "state, citizenship and democracy on the internet ?. anal.polit. [Online]. 2010, vol.23, n.68, pp.3-25. ISSN 0121-4705.

14. Mayorga L. and Galaviz G. (2017) The importance of information technologies in the public sector Available at: https://www.auren.com/es-MX/blog/sectorpublico/2017/01/ 16 / the-importance-of-information-technologies-in-the-publicsector

15. Law on Government. Official Gazette No. 40,274. October 17, 2013. 
16. Organic Law of Science, Technology and Innovation (LOCTI) Official Gazette No. 38,242 of August 2005

17. Law on data messages and electronic signatures (2001) Official Gazette No. 37.076, February 10, 2001

18. Telecommunications Law (2000) Gazette. Official No. 36,970. June 12, 2000

19.Palm, F. and Petrizzo, María A. (2010) Ways of Citizen Learning: Political Deliberation on the Internet. in Systems Think and e- Participation: ICT in the governance of society. Pachón-Cordoba, José (ed). United Kingdom: ICI Gobal

20.Palm, F. and Petrizzo, María A. (2010) Ways of Citizen Learning: Political Deliberation on the Internet. in Systems Think and e- Participation: ICT in the governance of society. Pachón-Cordoba, José (ed). United Kingdom: ICI Gobal

21. Perozo R and Marín A. (2019) Incidents of Web 2.0 Technology in the context of governance and governability. Iustitia Socialis. Arbitrated Journal of Legal Sciences. Year IV Vol. IV. No. 6 January - . June 2019. Made the deposit of Law: FA2016000064. ISSN: 2542-3371. KOINONIA FOUNDATION (F.K). Santa Ana de Coro, Venezuela

22. National Science and Technology Plan for Innovation (2005-2030) http://canaimaeducativo.me.gob.ve/catalogo-unico/contenidos/lecturassugeridas/plan-nacional-de-ciencia-tecnologia-e-innovacion- 2005-2030.pdf

23. Simón Bolívar National Plan 2001-2013. Official Gazette 6.295 Extraordinary May 1,2017

24. Ramírez T. (1999). How to do a Research Project. . Caracas Editorial Panapo.

25. Bolivarian Republic of Venezuela (2016) National Voluntary Presentation (PNV) to the United Nations High Level Political Forum on Sustainable Development (FPAN).

Available

at https://sustainabledevelopment.un.org/content/documents/10527Venezuela\%20PNV\%20DEFINITIVO\%20JUNIO\%202016\%20\%20DEFINITIVO\%20170620 $16 \% 20(1) \cdot \mathrm{pdf}$

26. SITEAL (2018). Educational Trends Information System in Latin America. Venezela National Telecommunications, Computer and Postal Services Plan (PNTIySP, 2007-2013) 
Iustitia Socialis. Revista Arbitrada de Ciencias Jurídicas.

Año IV. Vol. IV. Nº 6 . Enero - Junio 2019

Hecho el depósito de Ley: FA2016000064

ISSN: $2542-3371$

FUNDACIÓN KOINONIA (F.K). Santa Ana de Coro, Venezuela

Nathaly Serrano de Barrios

27. Shava, E. and C Hofisi, C. (2017) Challenges and Opportunities for Public Administration in the Fourth Industrial Revolution. Available at https://www.researchgate.net/profile/Elvin Shava2/publication/322676701 Challe nges and Opportunities for Public Administration in the Fourth Industrial Re volution/links/5a6865ada6fdcc03e0777a36/Challenges-and-Opportunities-forPublic-Administration-in-the-Fourth-Industrial-Revolution.pdf

(C2019 por el autor. Este artículo es de acceso abierto y distribuido según los términos y condiciones de la licencia Creative Commons Atribución-NoComercial-Compartirlgual 4.0 Internacional (CC BY-NCSA 4.0) (https://creativecommons.org/licenses/by-nc-sa/4.0/). 